\title{
Poynting vectors of Pc 5 pulsations observed by the GEOTAIL satellite in the dayside outer magnetosphere
}

\author{
Tohru Sakurai ${ }^{1}$, Yutaka Tonegawa ${ }^{1}$, Yuichi Shinkai ${ }^{1}$, Kiyohumi Yumoto ${ }^{2}$, Susumu Kokubun ${ }^{3}$, \\ Koichiro Tsuruda ${ }^{4}$, and Toshifumi Mukai ${ }^{4}$ \\ ${ }^{1}$ Department of Aeronautics and Astronautics, School of Engineering, Tokai University, 1,117 Kitakaname, Hiratsuka 259-1292, Japan \\ ${ }^{2}$ Department of Earth and Planetary Sciences, Kyushu University, 6-10-1 Hakozaki, Fukuoka 812-8581, Japan \\ ${ }^{3}$ Solar-Terrestrial Environmental Laboratory, Nagoya University, 3-13 Honohara, Toyokawa 442-8507, Japan \\ ${ }^{4}$ The Institute of Space and Aeronautical Science, 3-1-1 Yoshinodai, Sagamihara 229-8510, Japan
}

(Received April 6, 2000; Revised April 2, 2001; Accepted April 27, 2001)

\begin{abstract}
Poynting vectors of Pc 5 pulsations observed in the dayside outer magnetosphere are examined with the magnetic and electric field data simultaneously observed by the GEOTAIL satellite. Poynting energy can be estimated as about $10^{-8}-10^{-6} \mathrm{~W} / \mathrm{m}^{2}$ in both directions across and along the magnetic field-line. However, the energy propagating across the magnetic field-line is larger than the energy propagating along the magnetic field-line. From this result we can estimate the Pc 5 wave energy flowing into the inner magnetosphere during an hour as $10^{10}-10^{13} \mathrm{~J}$, which is one or two orders less than the substorm energy (about $10^{14}-10^{15} \mathrm{~J}$ ). However, by taking into account of the continuous activation of Pc 5 pulsations in the outer magnetosphere, Pc 5 waves should play an important role for energetics in the magnetosphere.
\end{abstract}

\section{Introduction}

Studies on Pc 5 pulsations by satellites in space suggested that they could be generated by the solar-wind driven surface waves at the magnetopause (Kokubun et al., 1976; Yumoto et al., 1983; Anderson et al., 1990). This idea has been supported by the field-line resonance theory from Chen and Hasegawa (1974) and Southwood (1974), and recently by a computer simulation on the Kelvin-Helmholtz instability (KHI) (Miura, 1984, 1987, 1990).

However, energetics on Pc 5 pulsations has not been investigated, except for a few papers (Greenwald and Walker, 1980; Junginger, 1985; Junginger et al., 1985; Chi and Russell, 1998). Greenwald and Walker (1980) studied ionospheric electric field oscillations and latitudinal resonance structures of transverse Pc 5 oscillations by using the Scandinavian Twin Auroral Radar Experiment (STARE) system. They found that the resonance wave energy in the magnetosphere was approximately $10 \%$ of the energy stored in the ring current.

On the other hand, Junginger et al. (1985) statistically investigated Poynting vectors of Pc 5 pulsations observed at the geosynchronous orbit by using the electric and magnetic field data measured from the electron-beam experiment and the magnetometer on board the GEOS-2 satellite. They found that the Poynting fluxes were measured in the range from $10^{-10}$ to $10^{-5} \mathrm{~W} / \mathrm{m}^{2}$. Most of the fluxes lay in the range from $10^{-9}$ to $10^{-7} \mathrm{~W} / \mathrm{m}^{2}$.

In this paper we will study Poynting flux of Pc 5 pul-

Copy right (C) The Society of Geomagnetism and Earth, Planetary and Space Sciences (SGEPSS); The Seismological Society of Japan; The Volcanological Society of Japan; The Geodetic Society of Japan; The Japanese Society for Planetary Sciences. sations observed in the outer magnetosphere. It was very fortunate that the GEOTAIL satellite surveyed successively in the outer magnetosphere. Several characteristics of Pc 5 pulsations observed by GEOTAIL near the flanks have been recently reported by Sakurai et al. (1999a, b), Tonegawa et al. (1997), Nakamura et al. (1994) and Kokubun et al. (1994). These studies revealed that large amplitude Pc 5 pulsations with transverse and compressional oscillations are frequently observed in the dawn- and duskside outer magnetosphere.

In order to know in detail how much energy is carried into the inner magnetosphere by the propagation of Pc 5 pulsations. Simultaneous observations of the magnetic and electric field data from GEOTAIL are analyzed as described in Section 2. In Section 3 a case study for a typical Pc 5 pulsation with transverse oscillations observed in the dawnside flank and its Poynting vector are examined. In Section 4 Poynting vectors for transverse Pc 5 pulsations observed both in the dawn and dusk flanks are statistically studied. Summary and discussions are given in the last section.

\section{Data and Analysis Procedure}

Data used in this study are the magnetic and electric fields measured by GEOTAIL when the satellite traversed near the dayside outer magnetosphere. The period of the Pc 5 data dealt in this study is covered 12 months through a year of 1995, from which we could examine on the 15 events of typical transverse Pc 5 oscillations.

The instruments and data processing techniques were described in detail for the magnetic and electric fields by Kokubun et al. (1994) and Tsuruda et al. (1994), respectively. In the present analysis we used $3 \mathrm{sec}$ average mag- 
netic and electric field data. Each field is transformed from the satellite coordinate to the mean-magnetic field coordinate. The mean-magnetic field is determined along with the three components of the magnetic field data for the duration of a Pc 5 event.

Mean field-aligned coordinates are very useful to examine oscillation characteristics with respect to the magnetic field direction, where $\boldsymbol{e}_{z}$ is in the direction of the mean magnetic field; $e_{y}$ is parallel to $e_{z} \times r$, where $r$ is a position vector of the satellite relative to the center of the Earth; and $\boldsymbol{e}_{x}$ (radial) is given by $e_{x}=e_{y} \times e_{z}$. The parallel electric field $E_{z}$ is zero by an assumption of plasma frozen in the magnetic field, $\boldsymbol{E}+\boldsymbol{U} \times \boldsymbol{B}=0$.

Pc 5 events used in this study are determined by the following procedure. First the dynamic specrum of each component of the field was calculated. We found the portion where the Pc 5 spectrum continues over $30 \mathrm{~min}$, and the spectral power exceeds $5 \mathrm{~dB}$ over the background power, which we identify as a Pc 5 event in this study.

\section{A Typical Pc 5 Oscillation and Its Poynting Flux 3.1 A typical dawnside Pc 5 oscillation}

A typical dawnside Pc 5 oscillation is shown in Fig. 1 for an interval of an hour from 07:00 to 08:00 UT (07:17-08:01 MLT) on 14 January 1995 . The GEOTAIL satellite covered the L-value from 10.25 to 9.76 , and the magnetic latitude from $-8.52^{\circ}$ to $-9.47^{\circ}$. From the top to the bottom panels the three components of the magnetic field, i.e., the radial $\left(B_{x}\right)$, azimuthal $\left(B_{y}\right)$, and field-aligned $\left(B_{z}\right)$ components and the magnetic field intensity $\left(B_{t}\right)$, and the two components of the electric field, i.e., the $\operatorname{radial}\left(E_{x}\right)$ and azimuthal $\left(E_{y}\right)$ components. By surveying these field data we can see the large amplitude and long period oscillations with the period of about 10 min clearly in the radial magnetic field component $\left(B_{x}\right)$. The period is longer than that of the usual Pc 5 oscillations. These longer period oscillations are seen in all the other components of the magnetic field and in the field intensity.

Two or three pulses of Pc 5 oscillations are seen around $7: 45$ UT in the azimuthal component of the magnetic field $\left(B_{y}\right)$, which is superposed on the longer period oscillations. However, the Pc 5 amplitudes are small. Contrary to the magnetic field oscillations, the Pc 5 oscillations are clearly seen in the electric field. Especially in the $E_{x}$ component the oscillations of the Pc 5 are clearly identified. In the $E_{y}$ component the oscillations are not regular, but the oscillations are identified as the Pc 5. Therefore, we can conclude that the large amplitude Pc 5 oscillations are clearly seen in the $E_{x}$ component. Therefore, we can infer that this Pc 5 oscillation is an odd mode oscillation of the magnetic field-line, since the magnetic field-line behaves as an anti-node at the magnetic equator for this oscillation. This is a typical signiture of Pc 5 oscillations observed by the GEOTAIL satellite, which traverses near the magnetic equator, i.e. the magnetic latitude is about $-9^{\circ}$.

Figure 2 shows the power spectra of the three components of the magnetic field given in the top panel, and the two components of the electric field in the bottom panel for an interval of $40 \mathrm{~min}$ from 07:20 to 08:00 UT. These spectra are calculated by using an autoregressive (AR) method which
An Example of Pc 5 Pulsations Observed by Geotail
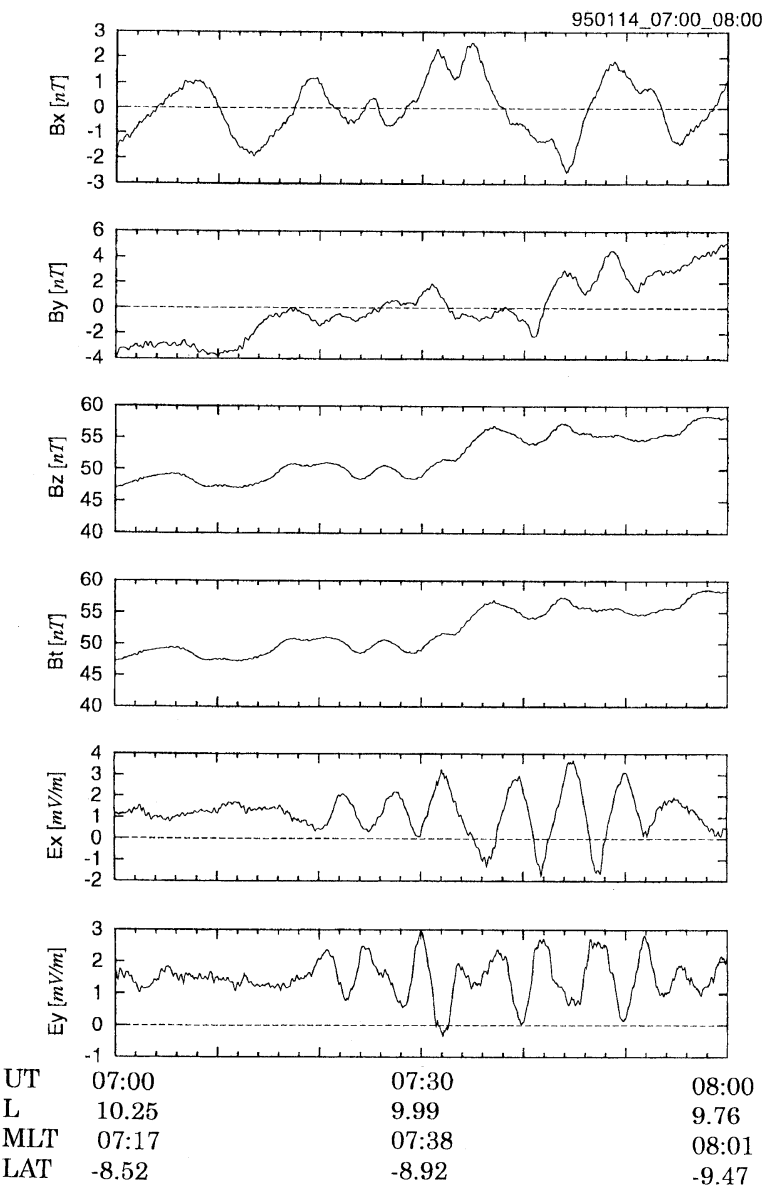

Fig. 1. A typical example of Pc 5 pulsations observed on 14 January 1995 in the dawnside outer magnetosphere by GEOTAIL. From the top to the bottom panels three components magnetic field $\left(\delta B_{x}, \delta B_{y}\right.$ and $\left.\delta B_{z}\right)$ and two components of the electric field ( $\delta E_{x}$ and $\delta E_{y}$ ) of the Pc 5 pulsations. The coordinates are mean-field coordinates.

gives us a logarithmically equal frequency resolution in the low frequency Pc 5 range (Tonegawa et al., 1984). The spectra show a clear peak in the $\delta E_{x}$ and $\delta E_{y}$ components near the frequency about $3 \mathrm{mHz}$. The spectral power dominates in the $\delta E_{x}$ component with one order larger than that of the $\delta E_{y}$ component. There is a corresponding spectral peak almost at the same frequency in the $\delta B_{y}$ component. However, the spectral peak is more dominant in the electric field compared with that of the magnetic field. Therefore, this Pc 5 oscillation gives a signature of transverse oscillations in the electric field.

\subsection{Poynting flux of the dawnside Pc 5 oscillations}

In this section we examine the characteristics of Poynting flux of the Pc 5 oscillations. Figure 3 shows a representative illustration of three components of Poynting vector, the radial $\left(S_{x}\right)$, azimuthal $\left(S_{y}\right)$, and field-aligned $\left(S_{z}\right)$ components, and the polar angle $\mathrm{A}$ between the $\mathrm{Z}$ axis and the Poynting vector $S$, where the $Z$ axis is parallel to the mean magnetic field direction. The angle $\mathrm{B}$ is set as an azimuthal angle between the $\mathrm{X}$ axis and the $S_{\text {perp }}$ of the $S$. The $S_{\text {perp }}$ is a component of the $S$ perpendicular to the mean-magnetic field. 
Power Spectra of Pc 5 Pulsations
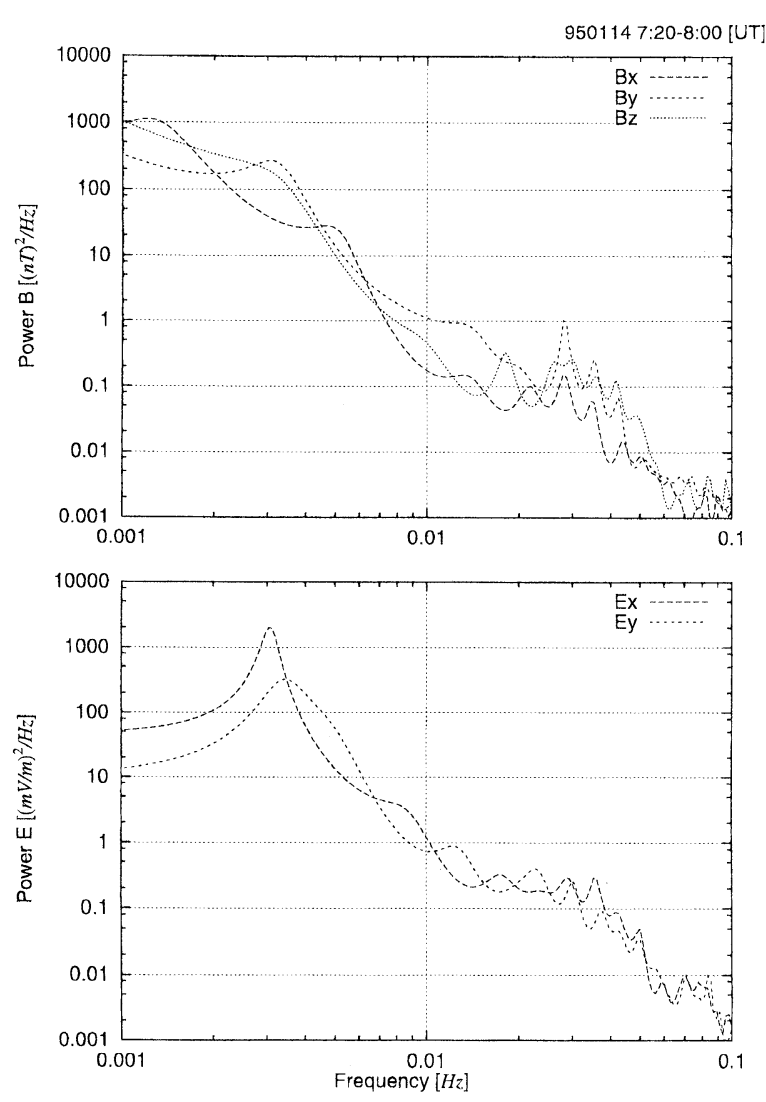

Fig. 2. Power spectra of the three components of the magnetic field (top panel) and of the two components of the electric field (bottom panel) of the Pc 5 pulsations shown in Fig. 1. The spectrum of $\delta E_{x}$ component is dominant. Suggesting that the Pc 5 oscillations are characterized with the transverse oscillations.

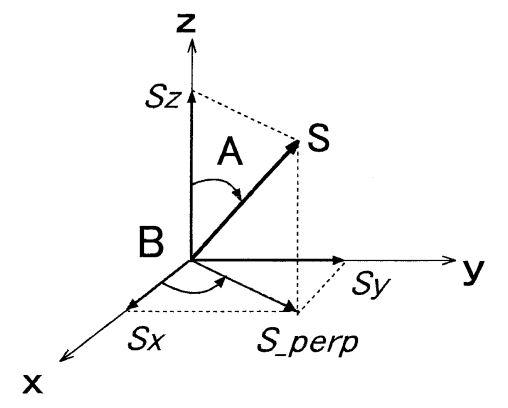

Fig. 3. The coordinate of Poynting vector $\boldsymbol{S}$ with respect to the mean magnetic field direction, $Z$. See details in the text.

Three components of the Poynting vector and the angles, $\mathrm{A}$ and B for the Pc 5 pulsations discussed in the previous Subsection 3.1 are plotted from the top to the bottom panels of Fig. 4. The Poynting flux is calculated with three components of the magnetic field perturbations of the Pc $5, \delta B_{x}$, $\delta B_{y}$ and $\delta B_{z}$, and the two components of the electric field perturbations, $\delta E_{x}$ and $\delta E_{y}$. They are band-pass filtered magnetic and electric field perturbations. The band-pass filter is designed with a frequency width of $3 \mathrm{mHz}$ from $2 \mathrm{mHz}$ to $5 \mathrm{mHz}$ centered at the frequency of $3 \mathrm{mHz}$. The field-
Poynting Flux

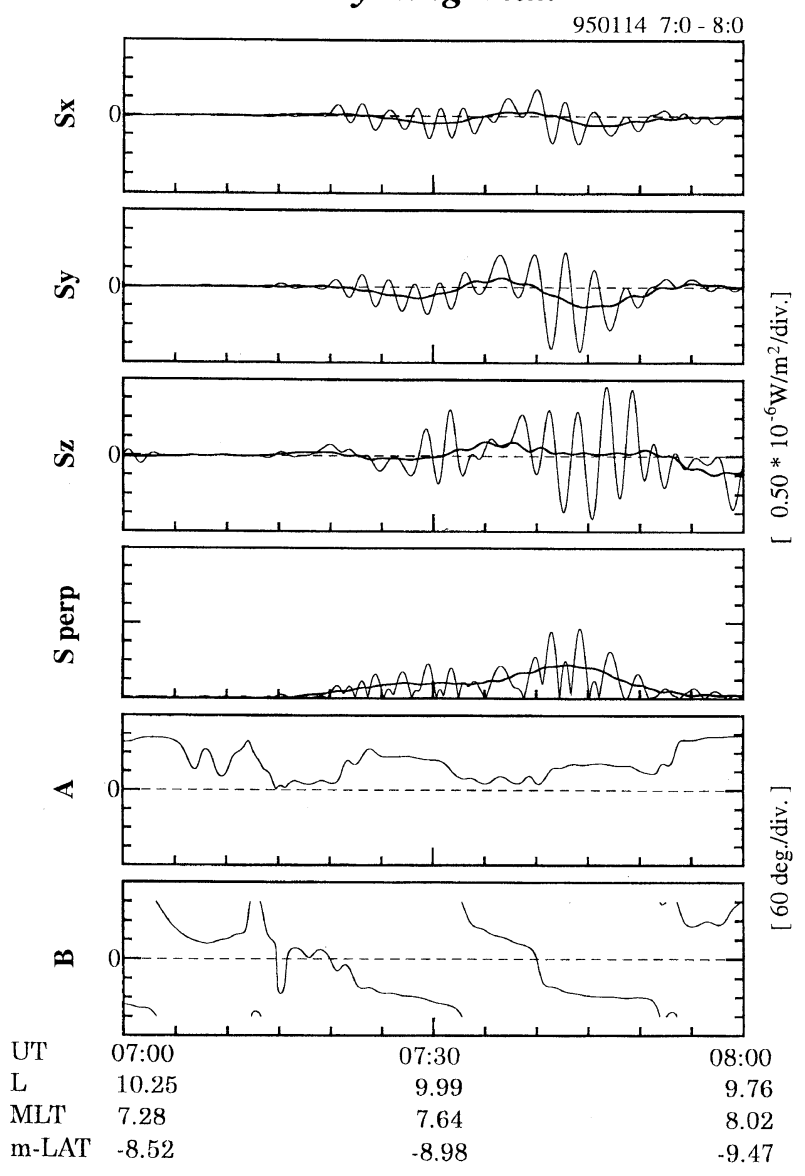

Fig. 4. Three components of Poynting vector $\boldsymbol{S}$ of the Pc 5 oscillations given in Fig. 1, the radial component $\boldsymbol{S}_{x}$, azimuthal component $\boldsymbol{S}_{y}$, and field-aligned component $\boldsymbol{S}_{z}$ from the top to the third panels, respectively. From the forth to the bottom panels show the perpendicular component $\boldsymbol{S}_{\text {perp }}$, to the mean-magnetic field, the polar angle A with respect to the Poynting vector $\boldsymbol{S}$ and the azimuthal angle B between the $\mathrm{X}$ axis and the $\boldsymbol{S}_{\text {perp }}$. The thick solid curves from the top to the forth panels show a smoothing value for a wave period.

aligned component of the electric field is assumed to be zero as described in Section 2. The calculation of the Poynting flux is performed using the formula of Poynting vector as follows;

$$
\boldsymbol{S}=\delta \boldsymbol{E} \times \delta \boldsymbol{B} / \mu_{0} .
$$

Each component of Poynting flux is calculated by using values sampled at each 3 second from both electric and magnetic fields and is represented in Fig. 4 with a thin solid line, which oscillates with a frequency double that of Pc 5 oscillations. While, the thick solid line represents the smoothing value of the Poynting flux averaged over a wave period $(T)$ according to the following equation,

$$
\langle\boldsymbol{S}\rangle=1 / T \int_{0}^{T} \boldsymbol{S} d t
$$

where a wave period $T$ is used with a representative period for the Pc 5 event by knowing the dominant spectral power as shown in Fig. 2, in which we can identify the dominant period of the Pc 5 as $T=330 \mathrm{sec}(f=3 \mathrm{mHz})$ in the $\delta E_{x}$ component. Surveying the average values of the Poynt- 
Table 1. List of Pc 5 pulsations.

\begin{tabular}{crrrrrrrr}
\hline M/D/Y & UT.s & UT.e & MLTs & MLTe & L-val.s & L-val.e & MLATs & MLATe \\
\hline 01/14/95 & $5: 10$ & $5: 45$ & 5.98 & 6.27 & 11.53 & 11.81 & -7.17 & -7.40 \\
$01 / 14 / 95$ & $6: 30$ & $7: 00$ & 6.93 & 7.28 & 10.54 & 10.25 & -8.09 & -8.52 \\
$01 / 14 / 95$ & $7: 20$ & $8: 00$ & 7.64 & 8.02 & 9.09 & 9.76 & -8.98 & -9.49 \\
$01 / 23 / 95$ & $9: 40$ & $10: 25$ & 7.99 & 8.73 & 9.99 & 9.76 & -6.92 & -7.45 \\
$03 / 20 / 95$ & $3: 30$ & $5: 00$ & 3.38 & 4.27 & 11.55 & 10.96 & -9.09 & -9.28 \\
$03 / 20 / 95$ & $6: 30$ & $8: 00$ & 5.23 & 6.22 & 10.70 & 10.60 & -9.13 & -8.32 \\
$03 / 25 / 95$ & $15: 00$ & $15: 30$ & 5.18 & 5.52 & 10.92 & 10.94 & -12.60 & -13.29 \\
$04 / 05 / 95$ & $9: 00$ & $9: 30$ & 5.61 & 5.93 & 10.40 & 10.44 & -5.65 & -5.25 \\
$04 / 10 / 95$ & $17: 00$ & $18: 00$ & 5.00 & 5.68 & 11.67 & 11.84 & -19.40 & -20.19 \\
$06 / 03 / 95$ & $8: 30$ & $9: 00$ & 3.54 & 3.81 & 10.56 & 10.72 & -2.65 & -1.97 \\
$06 / 03 / 95$ & $9: 00$ & $9: 30$ & 3.81 & 4.08 & 10.72 & 10.90 & -1.97 & -1.28 \\
$06 / 03 / 95$ & $9: 30$ & $10: 00$ & 4.08 & 4.34 & 10.90 & 11.10 & -1.28 & -0.59 \\
$06 / 08 / 95$ & $16: 30$ & $17: 30$ & 3.49 & 4.15 & 11.94 & 12.27 & -19.61 & -19.15 \\
$10 / 14 / 95$ & $21: 00$ & $22: 00$ & 17.83 & 18.15 & 10.38 & 10.43 & -5.87 & -5.38 \\
$11 / 15 / 95$ & $21: 00$ & $21: 30$ & 14.64 & 14.97 & 10.35 & 10.35 & -6.53 & -6.79 \\
\hline
\end{tabular}

ing flux the largest amplitude oscillations occur in the $S_{z}$ component, especially for the interval of about $10 \mathrm{~min}$ from 07:40 to 07:50 UT. This part corresponds to the period of the largest amplitude oscillations of a radial component of the electric field $\left(\delta E_{x}\right)$ of the Pc 5 oscillations. The average value, $\left\langle S_{z}\right\rangle$ shown with a thick solid curve is, however, very small and is close to the base line, suggesting that most of the wave energy bounces back and forth along the magnetic field-line. This means that the resonant oscillation is almost established in this part.

If we take the ratio of average Poynting energy parallel to the perpendicular one, i.e., $\left\langle S_{z}\right\rangle$ to $\left\langle S_{\text {perp }}\right\rangle$, it is almost zero. Therefore, most of the wave energy propagates across the magnetic field-line. In the fifth panel the angle $\mathrm{A}$ is about $90^{\circ}$. This value means that the energy propagates almost perpendicular to the magnetic field-line. The angle B in the bottom panel shows $-120^{\circ}$, indicating that the wave energy propagates azimuthally westward, i.e., tailward across the magnetic field-line. Therefore, in this part the wave energy propagates almost tailward perpendicular to the magnetic field-line.

Another interval showing a relatively clear oscillation takes place around 07:30 UT, where the average value of $\left\langle S_{z}\right\rangle$ is also close to the base line varying from the negative value to the positive one with a small amplitude. However, the $\left\langle S_{x}\right\rangle$ and $\left\langle S_{y}\right\rangle$ show a clear negative value. The $\left\langle S_{\text {perp }}\right\rangle$ shows a value of $\sim 5 \times 10^{-7} \mathrm{~W} / \mathrm{m}^{2}$. The angle $\mathrm{B}$ in the bottom panel shows also $-120^{\circ}$. These observed facts indicate that most of the wave energy for this portion propagates azimuthally westward across the magnetic field-line also.

\section{Statistical Examinations of Poynting Vectors of Pc 5 Observed in the Outer Magnetosphere}

We tried statistically to examine Poynting vectors of Pc 5 pulsations observed in the dayside outer magnetosphere. The period of the data sampling covers over one year from
January to December 1995. The typical transverse Pc 5 pulsations examined in this study are sampled for 15 events, which are listed in Table 1. The universal times, magnetic local times, L-values and magnetic latitudes corresponding to the starting and ending times of the event are shown from the second to the ninth column, respectively. The L-value covers from 9 to 12 . The 13 events are observed in the dawn sector and only two events are in the afternoon and dusk sectors, respectively.

Table 2 gives characteristics of the Poynting vector of the Pc 5 pulsations. The columns from the second to the forth show three components of the average values of the Poynting flux, $\left\langle S_{x}\right\rangle,\left\langle S_{y}\right\rangle$, and $\left\langle S_{z}\right\rangle$, respectively. They are given with a unit of $10^{-6} \mathrm{~W} / \mathrm{m}^{2}$. The positive and negative signs of $\left\langle S_{x}\right\rangle$ mean that the Poynting flux directs radially outward and inward at the satellite position, respectively. Similarly the positive and negative signs of the $\left\langle S_{y}\right\rangle$ mean that the energy propagates azimutally eastward and westward, respectively. The northward propagation of the field-aligned component, $\left\langle S_{z}\right\rangle$ shows with a positive sign, and the southward propagation with a negative one.

The fifth to seventh columns of Table 2 give the average Poynting flux perpendicular to the magnetic field-line, $\left\langle S_{\text {perp }}\right\rangle$, the polar angle, A and the azimuthal angle, B, of the $\left\langle S_{\text {perp }}\right\rangle$, respectively.

The eighth column shows a ratio of $\left\langle S_{z}\right\rangle$ to $\left\langle S_{\text {perp }}\right\rangle$, which means how much energy can propagate along the magnetic field-line compared with the energy propagating perpendicular to the magnetic field-line. From this column we can understand that for most of the events the ratio seems to be small with a few exception. However, the average value of the ratio of $\left\langle S_{z}\right\rangle$ to $\left\langle S_{\text {perp }}\right\rangle$ is 0.40 . This result means that the average energy propagating along the magnetic field-line is not so large. It is only $40 \%$ compared with the wave energy propagating across the magnetic field-line.

As shown in Fig. 5 we can plot the dominant propagating 
Table 2. Poynting fluxes of Pc 5 pulsations.

\begin{tabular}{cccccccrrr}
\hline $\mathrm{M} / \mathrm{D} / \mathrm{Y}$ & \multicolumn{1}{c}{$\left\langle S_{x}\right\rangle$} & \multicolumn{1}{c}{$\left\langle S_{y}\right\rangle$} & \multicolumn{1}{c}{$\left\langle S_{z}\right\rangle$} & $\left\langle S_{\text {perp }}\right\rangle$ & $\mathrm{A}(\mathrm{deg})$ & $\mathrm{B}(\mathrm{deg})$ & $\left\langle S_{z}\right\rangle /\left\langle S_{\text {perp }}\right\rangle$ & Ellipt. \\
\hline $01 / 14 / 95$ & -0.30 & -0.47 & 0.27 & 0.60 & 66 & -120 & 0.45 & 0.0 \\
$01 / 14 / 95$ & -0.06 & -0.05 & 0.08 & 0.08 & 45 & -139 & 1.00 & -0.2 \\
$01 / 14 / 95$ & -0.26 & -0.50 & 0.00 & 0.80 & 90 & -120 & 0.00 & 0.0 \\
$01 / 23 / 95$ & -0.17 & -0.52 & 0.26 & 0.52 & 64 & -109 & 0.50 & 0.1 \\
$03 / 20 / 95$ & -0.16 & -0.16 & 0.08 & 0.24 & 72 & -132 & 0.33 & -0.2 \\
$03 / 20 / 95$ & -0.67 & -1.33 & -0.27 & 1.73 & 81 & -113 & -0.16 & -0.3 \\
$03 / 25 / 95$ & -0.67 & -1.47 & 0.93 & 2.00 & 65 & -110 & 0.47 & 0.0 \\
$04 / 05 / 95$ & -0.13 & -0.40 & 0.13 & 0.80 & 81 & -99 & 0.16 & 0.0 \\
$04 / 10 / 95$ & -0.13 & -0.26 & -0.13 & 0.40 & 108 & -109 & -0.33 & -0.3 \\
$06 / 03 / 95$ & -0.06 & -0.06 & 0.03 & 0.13 & 77 & -117 & 0.23 & 0.1 \\
$06 / 03 / 95$ & 0.17 & 0.40 & 0.37 & 0.60 & 58 & 73 & 0.62 & -0.1 \\
$06 / 03 / 95$ & -0.96 & -1.49 & -1.49 & 1.81 & 129 & -122 & -0.82 & -0.2 \\
$06 / 08 / 95$ & -0.10 & -0.26 & -0.06 & 0.33 & 100 & -107 & -0.18 & -0.3 \\
$10 / 14 / 95$ & 0.06 & 0.26 & -0.60 & 1.20 & 116 & 87 & -0.50 & 0.2 \\
$11 / 15 / 95$ & 0.01 & -0.06 & -0.02 & 0.06 & 108 & 80 & -0.33 & 0.2 \\
\hline
\end{tabular}

Summary plots of the perpendicular component of the Poynting Flux, $<$ Sperp $>$

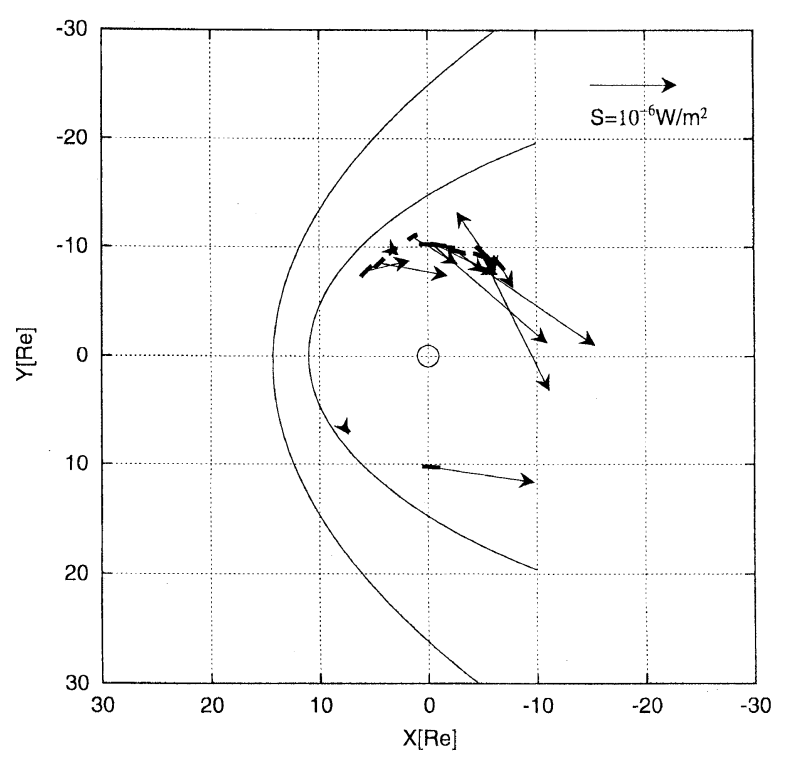

Fig. 5. A summary plot of the perpendicular component, $\left\langle\boldsymbol{S}_{\text {perp }}\right\rangle$ of the average Poynting vector for all Pc 5's dealt in this study. The samples are 15 cases of the Pc 5 pulsations. The arrows indicate the direction of $\left\langle\boldsymbol{S}_{\text {perp }}\right\rangle$.

direction of the average wave energy across the magnetic field-line by using the values of $\left\langle S_{\text {perp }}\right\rangle$ and B. The magnitude of the energy of each event and the corresponding azimuthal angle, $\mathrm{B}$ are given with an arrow. The plotted arrows show that in the dawn sector 12 cases show tailward propagation and only one case is radially outward. In the afternoon and dusk sectors each case is tailward. Although the event is not enough for a statistical examination, we can draw a conclusion from this figure that for most of the Pc 5 events the Poynting energy propagate tailward perpendicu- lar to the magnetic field-line in both flanks.

The average locations of the magnetopause and the bow shock are depicted with the solid curves based on the models given by Sibeck et al. (1991) and Fairfield (1971). It is clear that the Pc 5 pulsations occur only in the dawn and dusk sectors, extending from 9 to 12 in L-value in the dawnside, and near 10 in the duskside, respectively. They are observed fairly inside from the magnetopause. The ellipticity of these Pc 5 pulsations is tabulated in the last column and shows a very small value. The observed ellipticity also suggests that these Pc 5 pulsations show resonant oscillations at these places.

\section{Discussion and Conclusions}

In this paper we examined the Poynting flux of Pc 5 pulsations observed by the Geotail satellite during the 12 months from January to December 1995 . The analysis was performed by using the simultaneously observed electric and magnetic field data. A number of the study on Pc 5 pulsations so far based on the satellite data has been restricted to the magnetic field data only (Heppner et al., 1970; Hedgecock, 1976; Kokubun et al., 1976; Anderson et al., 1989, 1990; Cao et al., 1994). A very few satellite has provided the data of the electric field variations, such as DE1, ISEE's, and GEOS 2 satellites (Cahill et al., 1986; Chi and Russell, 1998; Junginger, 1985; Junginger et al., 1985). Therefore, our knowledge on the electric field signature of ULF waves in the magnetosphere has been substantially insufficient. The GEOTAIL satellite can provide both data of the electric and magnetic fields. In this study we examined with two different methods, one is a case study and the other is a statistical one. The results obtained are summarized as follows;

1) The average value of Poynting flux of Pc 5 pulsations are within an order of $10^{-6}$ to $10^{-8} \mathrm{~W} / \mathrm{m}^{2}$, and the largest one exceeds $1.0 \times 10^{-6} \mathrm{~W} / \mathrm{m}^{2}$. 
2) In a plane perpendicular to the magnetic field-line the wave energy propagates tailward. This signature can be seen in both dawn and dusk outer magnetosphere, though only two events are in the afternoon and dusk sectors.

3) The oscillating amplitude of the field-aligned Poynting flux, $S_{z}$ is large, and the oscillations of $S_{z}$ are symmetry with respect to the base line, suggesting that the wave energy bounces back and forth along the magnetic field-line, and the resonance oscillations are almost established there.

4) The average energy propagating along the magnetic field-line is not so large. It is only $40 \%$ compared with the wave energy propagating across the magnetic fieldline.

These results represent the signature of the Pc 5 Poynting flux observed in the outer magnetosphere. Comparing these results with the previous studies such as those by Junginger (1985) and Junginger et al. (1985). They have examined Poynting vectors of Pc 5 pulsations observed at the geosynchronous orbit by using the GEOS 2 satellite, and showed that Poynting vectors at the geosynchronous orbit were within the value from $10^{-5}$ to $10^{-10} \mathrm{~W} / \mathrm{m}^{2}$. The majority of them was in the value from $10^{-6}$ to $10^{-9} \mathrm{~W} / \mathrm{m}^{2}$. Thus, the values obtained at the geosynchronous orbit are found to be almost same to those obtained in our present study.

Another interesting conclusion obtained from this study is on the propagation direction of Poynting vector of Pc 5 across the magnetic field-line. Most of the events show the tailward propagation in both flanks. This is consistent with the results obtained by Junginger et al. (1985). The statistical results in their paper (Junginger et al., 1985) showed that the majority was consistent with the expected direction of propagation of solar-wind driven surface waves on the outer boundary of the magnetosphere.

Junginger (1985) has also examined Poynting vectors by using a computer simulation. Their results showed that the radial component of the Poynting vector is smaller than the azimuthal component for Pc 5 events observed between the magnetopause and the resonance region, where the azimuthal component is parallel to the phase velocity of the surface waves. Whereas on the earthward side of the resonance region the direction of the azimuthal component is antiparallel and directs to the nose of the magnetosphere. In our study most of the events shows the tailward propagation. This is also consistent with those obtained outside the resonance region by Junginger (1985). Moreover, our result shows that the ellipticity is almost linear, suggesting that the Pc 5 events dealt in our study occur near the resonant region.

Another computer simulation has been done by Miura (1984, 1987, 1990). Miura has applied his results to excitation of ULF waves observed in the outer magnetosphere and has clarified that for a realistic condition at the magnetopause a fastest growing mode has a wave period in the Pc 4 and Pc 5 range (45-600 sec). Most of the Poynting energy should propagate into the magnetosheath. As given in figure 6 in his paper Miura (1990) has revealed that the energy transmission into the magnetosphere by the KHI can be due to the kinetic energy only. However, the transmitted energy can be replaced with the Poynting flux inside the magnetosphere.

We studied Pc 5 pulsations observed in the outer magnetosphere. The energy of these Pc 5 pulsations is transported with the Poynting flux. Therefore, we can roughly estimate the energy flux propagating into the inner magnetosphere from the outside by using the Pc 5 Poynting flux obtained in the present study. As indicated in the item (1) the majority of the Poynting flux obtained in the outer magnetosphere is revealed to be in the value of $10^{-6} \sim 10^{-8} \mathrm{~W} / \mathrm{m}^{2}$. These values may correspond to a total energy of $10^{7} \sim 10^{9} \mathrm{~W}$, if we assume that the area of the flank should be $2 \times 10^{15} \mathrm{~m}^{2}$ by considering the longitude and latitude extents to be $90^{\circ} \times 20^{\circ}$ for the flank, although this is only an estimation.

Moreover, if an activity of Pc 5 pulsations continues for an hour on an average, the total energy transmitting into the inner magnetosphere should be $10^{10}-10^{13} \mathrm{~J}$. The largest value might correspond to $1 \sim 10 \%$ of the ring current energy $\left(10^{14}-10^{15} \mathrm{~J}\right)$.

If we take into account of the energy propagation along the magnetic field-line, it corresponds to the $40 \%$ of the energy propagation across the magnetic field. Therefore, this amount of the energy is expected to propagate to the ionosphere.

Greenwald and Walker (1980) have also investigated Poynting flux of the transverse Pc 5 pulsations observed in the ionosphere, based on the ionospheric ion drift measurements from the STARE system. The results were very indicative. The maximum value of Pc 5 electric field was observed to be $44 \mathrm{mV} / \mathrm{m}$, and its Joule heating energy was estimated to be $4.8 \mathrm{~mW} / \mathrm{m}^{2}$. The integrated total power of this Joule heating energy over the resonance region was found to be $6 \times 10^{9} \mathrm{~W}$. The corresponding total energy per an hour attains approximately $2 \times 10^{13} \mathrm{~J}$, which is the largest amplitude Pc 5 case in their study. However, the average Pc 5 amplitude is smaller than that of the largest one. If it is so, their results could be consistent with our expectation described above in the present study.

In conclusion there has been so far no examination on Poynting flux of Pc 5 measurements in the outer magnetosphere. Therefore, the examination of Poynting flux as was done in this study should be very oppotunate when we consider the importance of the propagation of the wave energy of Pc 5 in the dayside outer magnetosphere. The results are very suggestive not only to the propagation characteristics of Pc 5 but also to energetics in the magnetosphere, and for a causal relationship between the ionosphere and the magnetosphere. Thus, Pc 5 wave energy plays an important role for energetics in the magnetosphere and the ionosphere, when we consider the long lasting activation of Pc 5 oscillations in the outer magnetosphere.

Acknowledgments. Authors are indebted to all members of the GEOTAIL Project team. This work has not been done without much endeavor of the GEOTAIL Project team. Authors also sincerely acknowledge two anonymous reviewers for their critical readings of the manuscript and their constructive reviews. 


\section{References}

Anderson, B. J., M. J. Engebretson, and L. J. Zanetti, Distortion effects in spacecraft observations of MHD troidal standing waves: Theory and observations, J. Geophys. Res., 94, 13425-13446, 1989.

Anderson, B. J., M. J. Engebretson, S. P. Rounds, L. J. Zanetti, and T. A. Potemra, A statistical study of Pc 3-5 pulsations observed by the AMPTE/CCE magnetic experiment, 1. Occurrence distributions, J. Geophys. Res., 95, 10495-10524, 1990.

Cahill, L. J., Jr., N. G. Lin, M. J. Engebretson, D. R. Weimer, and M. Sugiura, Electric and magnetic observations of the structure of standing waves in the magnetosphere, J. Geophys. Res., 91, 8895-8908, 1986.

Cao, M., R. L. McPherron, and C. T. Russell, Statistical Study of ULF wave occurrence in the dayside magnetosphere, J. Geophys. Res., 99, 8731-8753, 1994.

Chen, L. and A. Hasegawa, A theory of long-period magnetic pulsations, 1. Steady state excitation of field line resonance, J. Geophys. Res., 79, 1024-1032, 1974.

Chi, P. J. and C. T. Russell, Phase skipping and Poynting flux of continuous pulsations, J. Geophys. Res., 103, 29479-29491, 1998.

Fairfield, D. H., Average and unusual locations of the earth's magnetopause and bow shock, J. Geophys. Res., 76, 6700-6717, 1971.

Greenwald, R. A. and A. D. M. Walker, Energetics of long period resonant hydromagnetic waves, Geophys. Res. Lett., 7, 745-748, 1980.

Hedgecock, P. C., Giant Pc 5 pulsations in the outer magnetosphere: A survey of HEOS-1 data, Planet. Space Sci., 24, 921-935, 1976.

Heppner, J. P., B. G. Ledley, T. L. Skillman, and M. Sugiura, A preliminary survey of the distribution of micropulsations in the magnetosphere from OGO's-3 and 5, Ann. Geophys., 26, 709-717, 1970.

Junginger, H., Poynting vector as a diagnostic of hydromagnetic wave structure, J. Geophys. Res., 90, 4155-4163, 1985.

Junginger, H., G. Haerendel, and F. Melzner, A statistical study of wave Poynting vectors measured during long-period magnetospheric pulsations at geostationary orbit, J. Geophys. Res., 90, 8301-8307, 1985.

Kokubun, S., R. L. McPherron, and C. T. Russell, Ogo 5 observations of Pc 5 waves, J. Geophys. Res., 81(28), 5141-5149, 1976.

Kokubun, S., T. Yamamoto, M. H. Acuna, K. Hayashi, K. Shiokawa, and H. Kawano, The GEOTAIL magnetic field experiment, J. Geomag. Geoelectr., 46, 7-21, 1994.

Miura, A., Anomalous transport by magnetohydrodynamic Kelvinhelmholtz instabilities in the solar wind-magnetosphere interaction, $J$.
Geophys. Res., 89, 801-818, 1984

Miura, A., Simulation of Kelvin-Helmholtz instability at the magnetospheric boundary, J. Geophys. Res., 92, 3195-3206, 1987.

Miura, A., Kelvin-Helmholtz instability for supersonic shear flow at the magnetospheric boundary, Geophys. Res. Lett., 17, 749-752, 1990.

Nakamura, M., H. Matsui, H. Kawano, A. Matsuoka, T. Yamamoto, K. Tsuruda, and H. Hayakawa, Pc 5 pulsations observed in the dayside magnetosphere by Geotail, Geophys. Res. Lett., 21(25), 2903-2906, 1994.

Sakurai, T., Y. Tonegawa, T. Kitagawa, M. Nowada, A. Yamawaki, T. Mukai, S. Kokubun, T. Yamamoto, and K. Tsuruda, Double-frequency oscillations of low energy plasma associated with transverse Pc 5 pulsations: GEOTAIL satellite observations, Earth Planets Space, 51, 41-53, 1999a.

Sakurai, T., Y. Tonegawa, T. Kitagawa, K. Yumoto, T. Yamamoto, S. Kokubun, T. Mukai, and K. Tsuruda, Dayside magnetopause Pc 3 and Pc 5 ULF waves observed by the GEOTAIL Satellite, Earth Planets Space, 51, 965-978, 1999b.

Sibeck, D. G., R. E. Lopez, and E. C. Roelof, Solar wind control of the magnetopause shape, location, and motion, J. Geophys. Res., 96, 5489$5495,1991$.

Southwood, D. J., Some features of field line resonance in the magnetosphere, Planet. Space Sci., 22, 483-491, 1974.

Tonegawa, Y., H. Fukunishi, T. Hirasawa, R. L. McPherron, T. Sakurai, and Y. Kato, Spectral characteristics of Pc 3 and Pc 4/5 magnetic pulsation bands observed near $L=6, J$. Geophys. Res., 89, 9720-9730, 1984.

Tonegawa, Y., T. Sakurai, T. Yamamoto, K. Tsuruda, and S. Kokubun, Spectral characteristics of Pc 3-5 pulsations observed by Geotail skimming the dayside magnetopause (Extended abstract), Proc. NIPR Symp. Upper Atmos. Phys., 10, 165-169, 1997.

Tsuruda, K., H. Hayakawa, M. Nakamura, T. Okada, A. Matsuoka, F. S. Mozer, and R. Schmidt, Electric field measurements on the GEOTAIL satellite, J. Geomag. Geoelectr., 46, 693-712, 1994.

Yumoto, K., T. Saito, and T. Sakurai, Local time asymmetry in the characteristics of Pc 5 magnetic pulsations, Planet. Space Sci., 31, 459-471, 1983

T. Sakurai (e-mail: sakurai@ms.u-tokai.ac.jp), Y. Tonegawa, Y. Shinkai, K. Yumoto, S. Kokubun, K. Tsuruda, and T. Mukai 